\title{
Violencia intrafamiliar en pacientes en edad pediátrica que recibe atención psicológica. Frecuencia, factores predisponentes y consecuencias
}

\author{
Cristina Haydée Arrom Suhurt ${ }^{\mathrm{I}}$, Andrés Claudelino Arce Ramírez ${ }^{\mathrm{I}}$, Carmen Marina Arrom Suhurt ${ }^{\mathrm{II}}$ \\ María del Pilar Fresco Arrom ${ }^{\mathrm{I}}$, Margarita Samudio', Marcos Capurro', María Auxiliadora Arrom de \\ Orrego $^{\text {II }}$, Mirta Mercedes Romero Núñez ${ }^{\mathrm{I}}$, Valentina Vargas ${ }^{\mathrm{I}}$ \\ I. Cátedra de Psiquiatría-Facultad de Ciencias Médicas de la Universidad Nacional de Asunción, Paraguay \\ II. Centro para el Desarrollo de la Investigación Científica CEDIC/Díaz Gill, Paraguay \\ III. Instituto de Investigaciones en Ciencias de la Salud-Universidad Nacional de Asunción IICS/UNA-Paraguay
}

Cómo referenciar este artículo/ How to reference this article:
Arrom C, Arce A, Arrom CM, Fresco MP, Samudio M, Capurro M et al. Violencia intrafamiliar en pacientes en edad pediátrica que recibe atención psicológica. Frecuencia, factores predisponentes y consecuencias. Mem. Inst. Investig. Cienc. Salud. 2015;13(3):24-30

\section{RE S U M E N}

El objetivo del estudio fue determinar la frecuencia y factores asociados a violencia intrafamiliar en pacientes en edad pediátrica. Estudio descriptivo de corte transversal en el que se incluyeron 119 pacientes en edad pediátrica que recibían atención psicológica en el Servicio Ambulatorio de la Cátedra de Psiquiatría de la Universidad Nacional de Asunción, Paraguay del 2010 al 2012. El 55,5\% $(n=66)$ de los 119 pacientes era del sexo femenino, $54,7 \%(n=65)$ tenía edades entre 12 y 18 años, y el $54,6 \% \quad(n=65)$ era residente de Asunción. El $74,8 \%(n=89)$ de los pacientes sufrió uno o varios tipos de violencia, siendo la violencia psicológica la más frecuente $(n=62,69,7 \%)$; seguida de violencia física $(n=57$, $64 \%)$. El abuso sexual se observó en 14 pacientes $(15,7 \%)$, el cual fue significativamente $(p<0,001)$ mayor en el sexo femenino. La violencia por abandono o negligencia que estuvo presente en 6 pacientes, fue mayor en los varones pero la diferencia no alcanzó significancia estadística $(p=0,08)$. La violencia entre padres se observó en 54 casos, la cual se asoció significativamente $(p<0,00001)$ con la violencia hacia los pacientes. En 82 pacientes se observaron problemas escolares, siendo su frecuencia mayor en aquellos víctimas de algún tipo de violencia pero no alcanzó significancia estadística $(p=0,08)$. Este estudio resalta la necesidad de intervenciones psico-educativas en servicios ambulatorios sobre las consecuencias de la violencia doméstica en la salud de estos pacientes para su desarrollo y salud física y mental. Además, son necesarios otros estudios que profundicen la temática para intervenciones prontas y oportunas.

Palabras clave: prevalencia, violencia intrafamiliar, niñez y adolescencia

\section{Domestic violence in a pediatric population receiving psychological care. Frequency, predisposing factors and consequences}

\section{A B S T R A C T}

The objective was to determine the frequency of domestic violence and associated factors in a pediatric population. This descriptive and cross-sectional study included pediatric patients receiving psychological care at the Outpatient Service of the Department of Psychiatry of the National University of Asuncion, Paraguay between 2010 and 2012. One hundred and forty five clinical records were studied. The $55.5 \%$ (66) was women, $7 \%$ (65) was between 12 and 18 years old and 54.6\% (65) was from Asuncion. The $74.8 \%$ 
(89) suffered one or more types of violence being the psychological violence the most frequent $69.7 \%$ (62) followed by physical violence 64\% (57). Sexual abuse was observed in 14 patients $(15.7 \%)$ which was significantly high in women $(p<0.001)$. Violence by abandonment or neglect was present in 6 patients and higher in male patients but the difference was not significant $(p=0.08)$. Violence between parents was found in 54 cases which was significantly associated with violence towards the patients $(p<0.00001)$. In 82 patients, school problems were observed being its frequency higher in those who were victims of some type of violence but it was not significant $(p=0.08)$. This study highlights the need for psycho-educational interventions in outpatient services on the consequences of domestic violence on the health of these patients for their physical and psychological development. Besides, other studies are necessary to deepen in this area for prompt and timely interventions.

Keywords: prevalence, domestic violence, childhood and adolescence

\section{INTRODUCCIÓN}

Según las Naciones Unidas, en América Latina entre $80 \%$ y $98 \%$ de niños y niñas sufren castigos corporales en el hogar, lo que vulnera sus derechos, afecta su desarrollo, salud y calidad de vida y sólo una pequeña proporción de estos actos son denunciados e investigados (1). La Convención Internacional sobre los Derechos del Niño (CIDN) (2) define al maltrato infantil como: "toda violencia, perjuicio o abuso físico o mental, descuido o trato negligente, malos tratos o explotación, mientras que el niño se encuentre bajo la custodia de sus padres, de un tutor o de cualquier otra persona que le tenga a su cargo".

El estudio de las Naciones Unidas sobre "Violencia contra Niños, Niñas y Adolescentes" tiene como lema central que ninguna forma de violencia contra los niños y niñas es justificable y toda violencia es prevenible. En el mismo se señala que para algunas culturas el castigo físico es tolerado y se requiere de estrategias nacionales de prevención con la adopción de medidas administrativas, legislativas y financieras para proteger a niños y niñas de todas las formas de violencia así como la creación de sistemas nacionales de recopilación de datos desagregados y fiables (1).

En Paraguay, el observatorio de Políticas Públicas y Derechos de la Niñez y la Adolescencia presentó un informe respecto a altos niveles de violencia intrafamiliar o en el hogar analizado a partir de 618 notas clasificadas por medios de comunicación, donde el $48,6 \%$ refirió violencia en el ámbito privado, hogar o domicilio. (3).

Se destaca que en Paraguay 6 de cada 10 niños y niñas en edad escolar reportaron algún tipo de maltrato en el hogar, en familias de diferentes estratos sociales y niveles de acceso a la educación, probando ser una práctica extendida y ampliamente justificada (3). Con relación a los casos de abuso sexual de niños y niñas se estima que se cuenta con registros limitados debido al temor de los afectados/as para realizar denuncias y además por la complejidad de los procedimientos que implican (1). Según denuncias realizadas ante la Fiscalía y presentados por la Secretaría Nacional de la Niñez y la Adolescencia un total de 421 niños y adolescentes fueron víctimas de abusos sexuales hasta el mes de mayo del 2015 en Paraguay (4).

Si bien las consecuencias de la violencia contra niños y niñas varían según factores de naturaleza y gravedad, sus consecuencias a mediano y largo plazo son poco difundidas. La exposición durante la primera infancia puede producir afecciones cerebrales en el proceso de maduración y la exposición prolongada como víctimas o testigos puede alterarles el sistema nervioso, provocar trastornos sociales, emocionales y cognitivos, además de otras conductas que causan enfermedades, lesiones y problemas sociales (5). Además se pueden citar otras consecuencias como el abuso de substancias, actividad sexual precoz, problemas de salud mental como trastornos de ansiedad y depresivos, deterioro en rendimiento laboral, alteraciones de la memoria y comportamientos agresivos. Finalmente el costo económico de este problema resulta muy alto a nivel de los países como a escala mundial (5).

Se tiene poco conocimiento de estudios que aborden con profundidad la justificación sostenida del uso de la violencia por parte de los padres y madres responsables de la crianza, que siguen utilizando la violencia física principalmente como instrumento para disciplinar a sus hijos e hijas. El conocimiento sobre este tema ayudaría a establecer 
mejores estrategias de prevención de la violencia y maltrato infantil en diferentes ámbitos.

De manera a contribuir con los conocimientos en esta área se planteó analizar la prevalencia de la violencia intrafamiliar y otros factores asociados en niños, niñas y adolescentes consultantes del Servicio Ambulatorio de la Cátedra de Psiquiatría de la UNA.

\section{PACIENTES Y MÉTODOS}

El estudio es descriptivo de corte transverso de la población pediátrica que recibe atención psicológica en el Servicio Ambulatorio de la Cátedra de Psiquiatría. Se estudiaron todas las fichas de pacientes entre 3 y 18 años de edad que consultaron entre enero del 2010 y diciembre del 2012.

Se realizó la revisión retrospectiva de las fichas de pacientes que acudieron a solicitar atención psicológica por distintas sintomatologías. De un total de $119,74,8 \%$ (89) había padecido alguna forma de violencia o maltrato en el ámbito familiar y $25,2 \%$ (30) no lo tuvo. Se procedió posteriormente a analizar los distintos tipos de violencia padecida por el grupo de estudio buscando las asociaciones posibles con otras variables como género, presencia de violencia doméstica entre los padres, problemas escolares y rangos de edad.

Los datos en las fichas clínicas fueron completados por el equipo de psicólogas y médicos residentes. Fueron consignados datos sociodemográficos (edad, sexo, procedencia), impresiones diagnósticas en el Eje I y en el Eje II en base a criterios propuestos por el Manual Diagnóstico y Estadístico (DSM IV TR) de la Asociación Americana de Psiquiatría; además de las distintas formas que asume la violencia intrafamiliar que fue definida de acuerdo a la clasificación propuesta por el grupo de trabajo sobre maltrato infantil dependiente del Ministerio de Salud de Chile (6) en: violencia o maltrato físico "toda lesión física no accidental producto de castigo único o repetido de magnitud y características variables"; de tipo emocional entendido como "hostigamiento verbal, habitual de un niño a través de insultos, criticas, descréditos y ridiculización, así como la indiferencia o rechazo implícito o explícito"; abandono o negligencia, considerado como "falta de protección y cuidados físicos al niño, de parte de sus figuras de custodia, así como el abandono de sus necesidades evolutivas por falta de estimulación cognitivas que no se deben a carencias extremas de recursos socioeconómicos"; y por último el abuso sexual "entre un niño y un adulto, aguda o crónica, incluida la explotación sexual".

Los datos fueron cargados en una hoja de cálculo Excel y posteriormente analizados con el paquete estadístico SPSS versión 15.0 para Windows 7. Los resultados se expresaron en forma de frecuencia absoluta y relativa; para indagar posibles asociaciones entre tipo de violencia y las demás variables se aplicó la prueba de chi cuadrado a un nivel de significancia de 0,05.

Se respetó la confidencialidad de la información asignando códigos a las fichas de los pacientes.

\section{RESULTADOS}

Fueron incluidos en el estudio 119 pacientes, más del 50\% tenía edades entre 12 y 18 años, $55,5 \%$ era del sexo femenino y $54,6 \%$ era residente de Asunción (Tabla 1 ). 
Tabla 1. Características socio demográficas de los pacientes en edad pediátrica consultantes del servicio ambulatorio de la Cátedra de Psiquiatría de la UNA. $n=119$

\begin{tabular}{llc}
\hline Características socio demográficas & $\mathbf{n}$ & $\%$ \\
\hline Grupo etáreo (años) & 15 & 12,6 \\
$1-5$ & 16 & 13,4 \\
$6-8$ & 23 & 19,3 \\
$9-11$ & 29 & 24,4 \\
$12-14$ & 36 & 30,3 \\
$15-18$ & & \\
Sexo & 66 & 55,5 \\
Femenino & 53 & 44,5 \\
Masculino & & \\
Procedencia & & \\
Asunción & 65 & 54,6 \\
Central & 39 & 32,7 \\
Interior & 14 & 11,8 \\
Exterior & 1 & 0,8 \\
\hline
\end{tabular}

El 74,8\% ( $n=89)$ de los pacientes sufrió algún tipo de violencia, de los cuales el más frecuente fue la violencia psicológica en 62 pacientes $(69,7 \%)$ seguida de violencia física en 57 casos (64\%) (Tabla 2).

Tabla 2. Tipo de violencia en los pacientes en edad pediátrica consultantes del Servicio Ambulatorio de la Cátedra de Psiquiatría de la UNA. $\mathrm{n}=89$

\begin{tabular}{lcc}
\hline Tipo de violencia & n & $\%$ \\
\hline Violencia psicológica & 62 & 69,7 \\
Violencia física & 57 & 64,0 \\
Abuso sexual & 14 & 15,7 \\
Violencia por negligencia o abandono & 6 & 6,7 \\
\hline Nota: un paciente pudo sufrir más de un tipo de violencia
\end{tabular}

El abuso sexual observado en 14 pacientes fue significativamente $(p<0,001)$ mayor en el sexo femenino $(26,5 \%)$ que en el masculino $(2,5 \%)$. La violencia por abandono o negligencia se observó en 6 pacientes y aunque fue mayor en varones no tuvo significancia estadística $(p=0,08)$ (Tabla 3$)$. 
Tabla 3. El género como factor predisponente de violencia en la población pediátrica

\begin{tabular}{llll}
\hline Género & $\mathbf{n}$ & $\begin{array}{l}\text { Abuso sexual } \\
(\mathbf{n = 1 4}) *\end{array}$ & $\begin{array}{l}\text { Negligencia o abandono } \\
(\mathbf{n = 6}) * *\end{array}$ \\
\hline Femenino & 49 & $13(26,5 \%)$ & $1(2,0 \%)$ \\
Masculino & 40 & $1(2,5 \%)$ & $5(12,5 \%)$ \\
\hline *Valor $\mathrm{p}=0,001 ; \operatorname{RR}(\mathrm{IC} 95 \%)=10,61(1,45-77,68)$ & \\
$* *$ Valor $\mathrm{p}=0,08 ; \mathrm{RR}(\mathrm{IC} 95 \%)=6,13(0,75-50,32)$ &
\end{tabular}

Si bien no se encontró asociación entre la edad y sufrir violencia, se pudo observar que la mayoría $(86,1 \%)$ de los adolescentes entre 15 a 18 años fue víctima de violencia (Tabla 4).

Tabla 4. Distribución de la población Víctima de violencia por Edad $(n=119)$

\begin{tabular}{llll}
\hline Edad (años) & n & Con violencia & Sin Violencia \\
\hline 3 a 5 & 15 & $11(73,3 \%)$ & $4(26,7 \%)$ \\
6 a 8 & 16 & $12(75,0 \%)$ & $4(25,0 \%)$ \\
9 a 11 & 23 & $16(69,6 \%)$ & $7(30,4 \%)$ \\
12 a 14 & 29 & $19(65,5 \%)$ & $10(34,5 \%)$ \\
15 a 18 & 36 & $31(86,1 \%)$ & $5(13,9 \%)$
\end{tabular}

Valor $\mathrm{p}=0,63$ (prueba chi-cuadrado)

La violencia doméstica del padre hacia la madre estuvo presente en 54 casos, y estuvo asociada significativamente a la violencia hacia los niños, niñas y adolescentes (valor $p$ $<0,00001$; prueba chi-cuadrado) (Tabla 5).

Tabla 5. Violencia doméstica como factor predisponente de violencia en la población pediátrica. $\mathrm{n}=$ 101

\begin{tabular}{lrrr}
\hline \multicolumn{1}{c}{$\begin{array}{c}\text { Violencia doméstica } \\
\text { entre padres }\end{array}$} & \multicolumn{3}{c}{ Violencia hacia paciente } \\
& $\mathbf{n} *$ & Si $(\mathbf{n = 7 4 )}$ & No $(\mathbf{n = 2 7})$ \\
\hline $\mathrm{Si}$ & 54 & $51(94,4 \%)$ & $3(5,6)$ \\
No & 47 & $23(48,9 \%)$ & $24(51,1 \%)$ \\
\hline $\begin{array}{l}\text { Valor } \mathrm{p}=<0,00001 ; \text { RR }(\mathrm{IC} 95 \%)=1,93(1,43-2,60) . \\
* 18 \text { pacientes fueron excluidos del análisis por no contar con datos }\end{array}$
\end{tabular}

Los problemas escolares se observaron en 82 pacientes, siendo ellos más frecuentes en aquellos que fueron víctimas de violencia $(79,7 \%$ vs $63,3 \%)$ pero la diferencia no alcanzó significancia estadística (valor p <0,08-prueba chi-cuadrado). (Tabla 6). 
Tabla 6. Violencia doméstica como factor predisponente de violencia en la población pediátrica. $n=$ 101

\begin{tabular}{cll}
\hline \multirow{2}{*}{ Violencia hacia paciente } & \multicolumn{2}{c}{ Problemas escolares } \\
& Si $(\mathbf{n = 8 2})$ & No $(\mathbf{n = 2 7})$ \\
\hline Si $(n=79 *)$ & $63(79,7 \%)$ & $16(17,7 \%)$ \\
No $(n=30)$ & $19(63,3 \%)$ & $11(36,7 \%)$ \\
\hline
\end{tabular}

Valor $\mathrm{p}=0,076$; RR: $1,26(0,94-1,69)$

*1 menor víctima de violencia sin escolaridad fue excluido del análisis.

\section{DISCUSIÓN}

Los niños, niñas y adolescentes del estudio han sido víctimas de violencia en un porcentaje elevado, principalmente maltrato físico y psicológico de parte de sus padres, tutores o encargados de la crianza y este estudio aporta evidencia de que, en el grupo de estudio al menos, continúan como práctica arraigada de forma de disciplinar distintas modalidades de violencia en el hogar, hecho ya evidenciado en estudios nacionales respecto al uso del castigo físico y otras formas en Paraguay (7-10). Las cifras expuestas se convierten en una realidad alarmante si se toma en cuenta que en estos hogares, otros menores estarían sufriendo la misma situación en calidad de testigos, presenciando estos hechos o padeciendo en calidad de víctimas, con graves consecuencias para su desarrollo posterior, salud y calidad de vida (11).

La clara asociación de violencia por razones de género expresada en el abuso sexual hacia niñas y adolescentes demuestra la persistencia de la profunda asimetría en el ejercicio abusivo de poder con agresión sexual hacia niñas, como lo señalan los estudios citados por UNICEF en el $2006(8,15)$. También señala que a la hora de ejercer sometimiento 0 castigos, padres, madres y encargados del cuidado utilizan preferentemente violencia psicológica hacia las niñas y adolescentes, mientras los varones fueron objeto de mayor abandono o negligencia. Estas prácticas muestran en forma determinante una marcada construcción social respecto a roles de género: aún se espera de las mujeres un rol pasivo, de obediencia y sometimiento, que requiere de una crianza con castigos que se correspondan con el objetivo, mientras que la libertad de cuidarse solos marca la tendencia a dejar a los varones con cierto riesgo negligente, por considerar que pueden arreglárselas sin tanto cuidado parental (16).

Se comprobó que en las familias donde se ejerció violencia hacia los niños, también se presentó violencia doméstica entre adultos, lo que coincide con lo evidenciado por la OMS (17). La violencia doméstica afecta finalmente a los demás miembros de la familia desde quienes detentan mayor poder hacia los más vulnerables. En este sentido, todos y todas las personas menores estarían expuestas como víctimas y testigos (17).

La población afectada por violencia de distinto tipo presentó problemas escolares y a pesar de que se observa una tendencia, con un número mayor en el tamaño de la muestra, se podría constatar la correlación que la bibliografía expresa como realidad.

Los resultados del estudio resaltan la necesidad de intervenciones psico-educativas para familias donde se presenta violencia hacia población infanto-juvenil. Los servicios ambulatorios ofrecen un buen espacio para trabajar con familias sobre las consecuencias de la violencia doméstica en la salud de niños, niñas y adolescentes. También enfocar las intervenciones sobre la valoración y legitimidad que goza la aplicación de castigos físicos como forma de disciplina familiar. Se requiere aumentar los estudios sobre violencia en el área hospitalaria por constituir un ámbito desde donde se puede intervenir en forma rápida y oportuna para orientar a las familias, diagnosticar los trastornos derivados de la violencia y tratarlos.

La violencia contra niños y niñas daña la salud física y mental a corto, mediano y largo plazo $(13,14,18)$, desestabiliza los entornos seguros de aprendizaje y hace estragos en la igualdad de género, es necesario protegerlos contra la violencia en todas las esferas de la sociedad y con ello estaríamos favoreciendo a los objetivos del desarrollo del Milenio en cuanto a una educación primaria universal y de paridad entre géneros, en reducir la mortalidad infantil y combatir el VIH/SIDA y otras enfermedades $(12,19)$. 


\section{REFERENCIAS BIBLIOGRAFICAS}

1. Naciones Unidas [Internet]. AsunciónParaguay: Movimiento Mundial por la Infancia y Secretaría Nacional de la Niñez y la Adolescencia; [citado 4 junio de 2013]. Disponible en: http://www.activamente.com.py/sites/defaul t/files/hoja_datos_sobre_violencia_en_parag uay.pdf

2. UNICEF. Convención sobre los Derechos del Niño. [Internet]. Nueva York-USA: Asamblea General de Naciones Unidas; [citado 14 junio 2014]. Disponible en: http://www.unicef.org/ecuador/convencion_ 2.pdf

3. PARAGUAY.com. Pobreza afecta al $45 \%$ de los niños paraguayos y la violencia al 61\%.[Internet]. Asunción-Paraguay: UNICEF; [citado 15 mayo 2015]. Disponible en:

http://www.paraguay.com/nacionales/pobre za-afecta-al-45-de-los-ninos-paraguayos-yla-violencia-al-61-82053

4. PARAGUAY.com. 421 niños abusados en 2015. [Internet]. Asunción-Paraguay: Secretaría Nacional de la Niñez y la Adolescencia (SNNA); [citado 15 junio 2014]. Disponible en: http://www.paraguay.com/nacionales/421ninos-abusados-en-2015-128572

5. UNICEF. Como eliminar la violencia contra los niños y niñas.[Internet]. Ginebra-Suiza: Unión Interparlamentaria (UIP), Oficina del Observador Permanente de la UIP, UNICEF: [citado 10 junio 2014]. Disponible en PDF: http://www.ipu.org/PDF/publications/violenc e_es.pdf

6. Poder Legislativo. Ley $N^{\circ}$ 1215/1986. Que aprueba la Convención sobre la eliminación de todas formas de discriminación contra la mujer. [Internet]. Asunción-Paraguay: Comité para la eliminación de todas las formas de discriminación contra la mujer (CEDAW)-Centro de Estudios Judiciales, 1986; [citado 20 junio de 2014]. Disponible en:

www.cej.org.py/desc/desc/.../agua/.../Ley\% 201215-1986\%20CEDAW

7. UNICEF. Estudio sobre maltrato infantil en el ámbito familiar en Paraguay [Internet]. Asunción-Paraguay: Base Educativa y Comunitaria de Apoyo (BECA), UNICEF; 2009. [citado 11 de junio 2013]. Disponible en PDF: http://www.unicef.org/paraguay/spanish/py _resources_Estudio_Maltrato.pdf

8. Arrom $C$, Ruoti $M$, Orué $E$, Arrom CM. El castigo y sus diferentes formas en la crianza de niños, niñas y adolescentes en 1049 familias de Alto Paraná. Revista Paraguaya de Psiquiatría. 2015;3(1):26-32.

9. Arrom $C$, Fresco MP, Arrom CM, Arce A, Arrom MA, Romero $M$, et al. Violencia intrafamiliar. Revista Paraguaya de Psiquiatría. 2015;3(1):38-41.
10. Ruoti M, Arrom C. Diferentes formas de violencia padecidas por adolescentes durante su crianza. Revista Paraguaya de Psiquiatría. 2015;3(1):50-6

11. Royal College of Psychiatrists. La violencia doméstica y sus efectos en el niño. [Internet]. London-England: Consejo Editorial de Educación Pública del Colegio Real de Psiquiatras; 2004 (actualizado de 2004, fecha de acceso 8 de Mayo 2014]. Disponible

en: http://www.rcpsych.ac.uk/healthadvice/tran slations/spanish/violenciadom\%C3\%A9stica. aspx

12. Fresco MP, Arrom C, Samudio M, Arrom CM, Capurro M, Arrom MA, et al. Psicopatología en adultos con antecedentes de violencia intrafamiliar. Revista Paraguaya de Psiquiatría. 2015;3(1):8-12.

13. Johnson J, Cohen P, Brown J, Smailes E, Bernstein D. Childhood maltreatment increases risk for personality disorders during early adulthood. Arch Gen Psychiatry 1999 Jul;56(7):600-6.

14. Battle CM, Shea $t$, Johnson DM, Yen S, Zlotnick C, Zanarini MC, et al. Childhood Maltreatment Associated with Adult Personality Disorders: Findings from the Collaborative Longitudinal Personality Disorders Study. Journal of Personality Disorders. 2004;18(2):193-211.

15. Secretario General de Naciones Unidas Derechos del niño.[Internet]. Santiago de Chile: Agencias de Naciones Unidas, Fondo de las Naciones Unidas para la Infancia (UNICEF); [citado 6 de junio de 2014]. Disponible en: http://www.unicef.org/violencestudy/reports /SG_violencestudy_sp.pdf

16. Instituto Nacional de las Mujeres. Roles del género. [Internet]. México-México: Instituto Nacional de las Mujeres, Dirección Estadística; [citado 6 de junio de 2014]. Disponible en: http://iknowpolitics.org/sites/default/files/dc to202.pdf

17. García-Moreno C, Guedes A, Knerr W. Comprender y analizar la violencia contra las mujeres. Violencia ejercida contra la pareja. [Internet]. Washington DC:OPS-OMS; 2013

18. Vitriol V, Gomberoff $M$, Basualto ME, Ballesteros S. Relación entre trastorno por estrés portraumático de inicio tardío, abuso sexual infantil y revictimización sexual. Caso clínico, Rev Méd Chile 2006;134:1302-5.

19. Naciones Unidas. Objetivos del desarrollo del milenio. Informe 2013. [Internet]. Nueva York-USA: Naciones Unidas; [citado 16 de junio de 2014]. Disponible en: http://www.un.org/es/millenniumgoals/pdf/ mdg-report-2013-spanish.pdf 